\title{
The Study of Virtual Reality Scene Making in Digital Station Management Application System Based on Unity3D
}

\author{
Jianren Wang ${ }^{1, a}$, Fushuan Wu ${ }^{1, \text { b }}$, Jie Wang ${ }^{2, c}$ \\ ${ }^{1}$ Wuhan Mechanical Technology College, Wuhan, 430075, China \\ ${ }^{2}$ Hebei University of Technology ,Langfang, 065000,China \\ a3230357068@qq.com, b1601286697@ qq.com, ${ }^{\text {c}} 815418241 @ q q . c o m$
}

Keywords: Unity3D; Digital Station; Virtual Reality.

\begin{abstract}
Combined with the application of Unity3D engine in the south-polar Digital Station Management Application System project, this paper introduced making station virtual reality scenes with Unity3D, and studied the critical technique of making virtual realityscenes, which contains importing real terrain station area, animation, scene lighting layout, correcting models material type, patticle system and external scene making.
\end{abstract}

\section{Introduction}

In recent years, the Virtual Reality technology is used more and more widely in all aspects, such as the military, information techonology, the real estate and so on. It generates the realistic virtual environment which is the multiple induction cosists of the sight, hearing ,touch and so on. It lets the users interact with the virtual environment's objects and make them feel immersive. And the Unity $3 \mathrm{D}$ as a three dimensional gaming platform of virtual reality, it can deal with plenty of three dimensional models adequately and in real time. This paper aims to use the Unity 3D engine, according to the Digital Station Management Application System to develop more projects and detailed introduces the critical techniques of virtual reality scenes when making the two stations--Changcheng Station and Zhongshan Station.

\section{The importing of the real terrain station area}

Unity 3D supports the importing of the real terrain station area, but it has the corresponding requirments about the surfaces and the mappings.

\subsection{The importing of the terrain models}

When the real terrain files of Changcheng Station and Zhongshan Station in the 3D Max importing to the Unity 3D engine, we find that the terrain files can't correctly transform to the Unity 3D's terrain system, for the Unity 3D software's maximum loading limitation to the triangle faces of the independent model is 65535, so it must go back to the 3D Max to modify the terrain files of the Changcheng Station and Zhongshan Station and reduce the surfaces to make it meet the requirements. Once again importing the terrain files, it can import correctly and transform to the Unity 3D terrain system and come to the terrain making process.

\subsection{The dispose of the terrain mappings}

Though the Unity 3D terrain system's mappings support several mappings overlapping display, after the terrain files are imported, it can let the station's images and photos laminate the real terrain. The Unity 3D does not support the original images which are too big, so it seriously affects the appearance of the terrain mappings. So in the production process, the terrain images are saved as JPEG format and reduce the image resolution, then they can import the terrain mappings successfully, but it will affect the quality of the station's surface imaging. 


\section{The implementation of the station animation}

\subsection{The station animation which is need to make}

The Changcheng and Zhongshan Stations' animation needs two softwares' cooperation to make it reality--the 3ds Max and the Unity 3D. It means that firstly, accomplishing the needing animation in the 3ds three dimensional production software, such as the animations of opening the door, the rotation of the switch and so on. Then let the animation's models import to the Unity 3D separately and can reset the parameters such as the animation's frames, names and so on. After the scenario runs, we can according to the needs and through the script controlling, decide how to play the animation, such as the door's clicking play.

\subsection{The played video aniation}

To make the scenario more vivid, it adds several video aniations in Changcheng and Zhongshan Station, such as the TV in life restaurant of Changcheng Station, it likes the real scenario and when clicking the telecontrol, the TV will open. And it can broadcast the BBC's documentaries of the south-polar penguins, as well as the news reports of the South Pole's research. In addition, there are the computer aniation of the Zhongshan Weather Stations, increasing the interests and authenticity of the scenario, as shown in figure 1 the TV is playing the documentaries of the south-polar penguins.

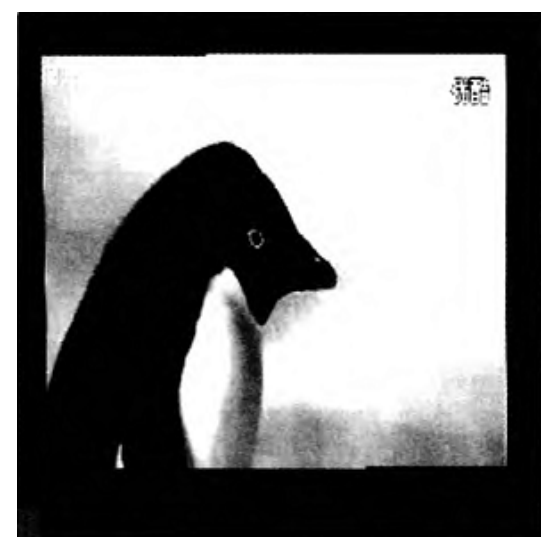

Fig.1 Penguin documentary on TV

\section{The scene lighting layout of the station}

Using the Unity 3D engine to make the senes of Changcheng and Zhongshan Station, the lighting is divided into two types, namely the outdoor lighting and the indoor lighting. The outdoor lighting refers to the light from the sun, also the Parallel light. To ensure the brightness of the whole scene, it sets up two parallel lights. One is normal and can produce shadows, the other does not have shadows and just the parallel light to increase the brightness of the scene. The indoor lighting refers to the light spot that layout inside the door to make the scene present more perfect. But to make the latter scene optimize, they should turn off the indoor lights of the scene in the daylight to save the system's resources.

\section{Correcting models material type}

For the two stations'models which are imported to the Unity 3D, the default models material type is mostly in the form of diffuse reflection, this type can't perfectly show the objects' features and effects. So the imported models must for the material type adjustment, as well as the Shader adjustment. The five common material types are the Normal, Transparent, Transparent-CutOut, Self-illumination and the Reflective.

1) Normal

For the untransparent texture objects, such as the diffuse reflection material.

2) Transparent

For the partly transparent objects, the texture’s alpha channel defines the transparency. 
3) Transparent-CutOut

To the objects which have the completely untransparent and completely transparent areas, such as the fence.

4) Self-illumination

For the self-illumination's objects, such as the signs of the corridor's safety exit.

5) Reflective

For the untransparent texture objects which can reflect the outside cube maps, such as the morror, itself is untransparent, but it reflects the outside world's maps.

\section{The Particle system}

\subsection{The production of the snow in the station}

The South-polar Changcheng Station and ZhongShan station's geographical position is special, the climate is cold and snowy. So it is necessary for the production of the snow in the station's weather system. The station uses Unity 3D's particle system to make snow, by regulating different weather's snow particles to show the variety weather of Changcheng and Zhongshan Station, and for this setting four snow types, including the light snow, moderate snow, heavy snow and the blizzard, and matches different wind effects. The figure 2 shows the weather condition of the light snow.

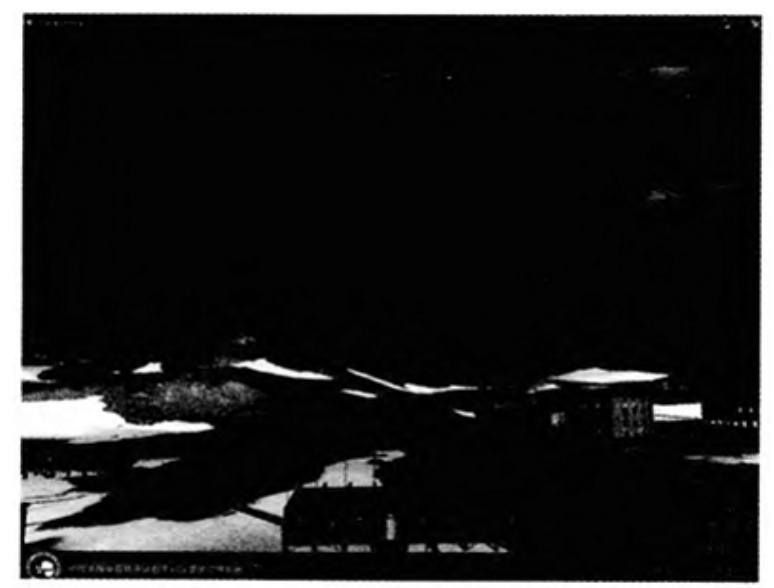

Fig.2 Great Wall Station snow weather condition

\subsection{The station's other particle systems}

The kitchen's water and fire of Changcheng and Zhongshan Station is also made by the particle system.

\section{The making of the external scene}

The making of the station's external scene including the scenes of the sky box, wind, fog, lake watre, ocean water and so on.

1) The sky box

Because this project realizes the founction of the station's switching of day and night, so it needs to make different sky boxes of different periods of time. Finally it produces different sky boxes including the daylight, dusk, night, cloudy day and so on.

2) The wind and fog

The late stages of the project additionally realizes the station's switching of different weathers. So to the South-Polar area whose geographical position is special, wind and fog are two necessary environmental factors of the scene. The wind and fog can adjust the parameters previously according to the scene's Render Settings, setting the values of the soft wind, breeze, strong wind, high wind and the mist, heavy fog, dense fog and so on, and realizing it by adjusting the scripts.

3) The lake water and ocean water

The Zhongshan Station's lake water and the Changcheng Station's ocean water all need Unity 
3D's built-in water system recources to make it realization, by the Parameter adjustment to fully shows the water resources of the station.

\section{Conclusion}

This paper based on the Unity 3D engine, by the practical experience of the Unity3D engine in the south - polar Digital Station Management Application System project, to study and summarize several key points of making virtual reality scenes. It still needs to make some improvements in the mapping clarity of the terrain system and the Overall beautification of the scene effect. In the follow-up work, it will make futher research on the application of the Unity 3D in the virtual reality scenes.

\section{References}

[1] L.B. Mi, P. Qi and L.N. Yi:The Sudy and Application of Virtual Display Tchnology Based on Unity3D (Digital Technology and Application), Vol. (2009) No. 12, p54-55. (In Chinese)

[2] Zh. Zhu:The Study of the Design and Application about the Virtual Experiment System Based on Unity3D (Ph.D.,Central China normal university,WuHan,China 2012), p.28.

[3] J.S. Gan:The Sudy of The Key Technology about Roaming in the Room Based on Unity3D(Journal of yancheng institute of technology) Vol24. (2011) No. 4, p56-59 (In Chinese)

[4] Guowei liang, Yuan Bo. Theory of virtual reality technology to create network immersion interactive art space [C]. Academic exchanges, 2010.12.

[5] Li Qin. Morphological characteristics of immersion transmission study [J]. Modern communications, 2013 (2). 